\title{
Understanding Women's Anxiety and Uncertainty attending a Rapid Diagnostic Clinic for Suspicious Breast Abnormality: A Mixed Methods Study
}

Christine Maheu ( $\nabla$ christine.maheu@mcgill.ca )

McGill University https://orcid.org/0000-0001-8704-8207

Bridgette Lord

University of Toronto

Christine Wang

University of Toronto

Ai Tanimizu

University of Toronto

David R. McCready

University Health Network

Jacqueline Galica

Queen's University, Kingston

Doris Howell

University of Toronto

\section{Research article}

Keywords: diagnosis, cancer, breast, uncertainty, anxiety, rapid diagnostic centre, nurse, psychological support, abnormality, oncology

Posted Date: November 27th, 2019

DOI: https://doi.org/10.21203/rs.2.17781/v1

License: (c) (1) This work is licensed under a Creative Commons Attribution 4.0 International License.

Read Full License

Version of Record: A version of this preprint was published at McGill Journal of Medicine on April 22nd, 2022. See the published version at https://doi.org/10.26443/mjm.v20i1.844. 


\section{Abstract}

Background: Rapid diagnostic centres (RDC) for breast abnormality offer a speedier process from the discovery of a suspicious breast lump to same-day investigation and confirmation of a breast cancer diagnosis. Study purpose: We aim to assess the anxiety and uncertainty levels of women going through an RDC and explore women's needs for support during the diagnostic period. Methods: Thirteen women who attended an RDC in 2014 took part in a sequential mixed-method study to assess anxiety and uncertainty levels. Measures were taken at pre and post-testing, at three weeks following receipt of results followed by a semi-structured telephone interview. Results: The mixed data results mainly showed congruence between women scoring high on anxiety and uncertainty levels and their detailed descriptions of the experience as stressful. Overall, uncertainty and anxiety levels were above the clinical threshold while awaiting further testing. These levels remained high only for the subgroup found with cancer and remained high at the three-week mark. As for the benign group, anxiety was above cut-off before diagnosis $(n=6)$, decreased to below clinical cut-off three days following diagnosis $(n=0)$, and increased again above cut-off three weeks post-diagnosis $(n=2)$. Discussion and Conclusion: While speedier testing may reduce waiting times to obtain further tests, results suggest that the period leading up to the day of testing is anxiety-provoking and marked with intense uncertainty. Results suggest the potential role of nurses to support waiting for patients with education and resources. These results illustrate the need for further investigation into psychological support options at RDCs.

\section{Background}

During the diagnostic period, after a breast lump is discovered or a mammogram displays suspicious findings, women experience significant psychological distress including anxiety, uncertainty, and symptoms of acute stress reaction (1-4). A systematic review of thirty research studies with large sample sizes examining the presence of anxiety levels in the diagnostic period for women with suspected with breast cancer found that on average, there were 8-50\% that experienced high levels of anxiety (5). The high anxiety was also found to persist throughout the diagnostic evaluation until certitude was achieved through the establishment of the definitive diagnosis (6). Such high levels of anxiety can easily disrupt an individual's ability to maintain their everyday activities and are also documented as having a negative effect on the immune system (7-9). Pre-diagnostic anxiety is also observed to be a strong predictor of post-diagnostic anxiety that can negatively influence treatment outcomes in women with breast malignancies and lead to unfavourable behavioural changes in women with benign disease $(3,5)$. However, although the diagnostic phase can be marked with intense emotional distress, this phase is often overlooked in research $(2,10-12)$.

Rapid diagnostic centres (RDCs) or one-stop clinics were developed to improve the diagnostic process for women and offer same-day investigation and a quick turn-around for a diagnosis of breast cancer (same day to three days post-investigation) $(2,11)$. Conceptually, the idea of RDCs for women with suspicious breast abnormalities is attractive, especially to reduce wait-times to diagnosis (13). However, for the few studies that have investigated the psychological impact of rapid diagnostic testing for breast cancer, the 
consensus remains unclear as to what are its effects on patient anxiety, uncertainty, and stress $(2,3,14-$ 16) although current longitudinal evidence is pointing towards a positive association between symptomatic breast disease and psychological distress $(3,5,17)$. In addition, there is limited information on whether receiving psychological support during the rapid diagnostic process is needed and if so, in what forms (12). The objectives of this study were to address these outstanding gaps by measuring and describing the levels of anxiety and uncertainty as experienced by women undergoing rapid diagnostic testing for a suspicious breast abnormality before and after testing and by exploring women's expressed needs for support during the diagnostic period.

Theoretical model

The Mishel Uncertainty in Illness model guided this study (18). Mishel ((18), p. 225) defines uncertainty as "the inability to determine the meaning of illness-related events". Uncertainty arises when a person is unable to characterize an event because of insufficient cues. This inability may be especially acute in a complex situation, such as a diagnostic workup, where the patient is overwhelmed with an abundance of unfamiliar cues. When a situation is appraised negatively, uncertainty is viewed as a threat rather than an opportunity. In these instances, anxiety becomes a covariate of uncertainty. Thus, one can anticipate that a reduction in uncertainty could, in turn, lead to a reduction in anxiety $(3,19)$.

\section{Results}

The women who took part in the study had a mean age of 50 , had an average of two children, the majority were married $(n=8 / 13)$, university-educated $(n=8 / 13)$, and had a family history of breast cancer $(n=7 / 13)$. Among the 13 women, seven were found with a malignant tumour and six with a benign tumour.

At the pre-diagnostic period, 9 out of 13 women scored above the clinical cut-off for anxiety and all scored above normative values for uncertainty (normative values $=33.7$ ) with an average score of 60 (SD 12.9) on a range of 23-115 (see Table 1). A two-tailed Pearson correlation coefficient test between anxiety and uncertainty revealed a strong association of $0.757(p=0.003)$.

The triangulated results from the quantitative and qualitative data are separated by categories: high versus low anxiety and uncertainty; prior to and after testing to reveal themes that could help explain their emotional experience.

Pre-diagnosis I: Accounts of women whose scores were below the cut-off for anxiety

Two themes best describe the experiences of the women with high uncertainty but low anxiety $(4 / 13)$ : use of positive reinterpretation and support received by clinical staff.

Use of Positive Reinterpretation: The four women in this category, although experiencing high levels of uncertainty, maintained their anxiety level below the cut-off by viewing their situation in a more favourable light, as an opportunity. That is, whenever they worried about their upcoming test results, the 
women described using repetitive positive thoughts and holding on to the belief that their suspicious breast abnormality would probably be benign. They viewed their situation as positive: "it is just the healthcare team erring on the side of caution (Participant \#2)." The four women also described the use of calming self-talk to help reinterpret negative anxiety-provoking thoughts into positive thoughts "I am able to get through it. I can deal with this...I'm strong (Participant \#1)." Another woman described how she practiced letting go of a situation she had no control over and how that process left her feeling less anxious "so I said to myself until I have more information I am just going to leave it and not try to think about what it could be (Participant \#2)."

Support by clinical staff: All the four women in this category mentioned how the support and reassurance that they received from the clinical team helped them to remain calm: "it's most likely not cancer (Participant \#9)." The clinical nurses' explanations as to why further tests were needed were also mentioned as helpful in reducing the four women's uncertainty and anxiety.

Pre-diagnosis II: Accounts of women whose scores were above the cut-off for both anxiety and uncertainty

For the larger group of women whose scores were above clinical cut-offs for both the anxiety and uncertainty scales (9/13), two themes best describe their experiences: 1 ) additional testing generating uncertainty, and 2) the ability to maintain responsibilities.

Additional testing generating uncertainty: These women described their lack of understanding of the additional testing which was being carried out, and how the situation of additional testing led to much uncertainty. They also talked about feeling anxious while waiting for the test results from their additional testing. Notwithstanding the small sample of 13 , this finding is aligned with the association found with the quantitative measures of anxiety and uncertainty before receiving their diagnostic results $(p=0.003)$. One participant described her anxiety arising from a statement made by her health professional that the participant could not decipher the true meaning of "they want to do a biopsy to check on some cells... What exactly did she mean? I consider this a statement with no ending (Participant \#8)." To another woman, the feeling of uncertainty occurred because the necessity of additional tests made it hard for her to view the situation in any other way than "catastrophic" (Participant \#9).

Concerns over maintaining responsibilities: Women who feared the "worst-case scenario

Women who expressed fears and increased perception of being found with cancer described how they were affected by intrusive thoughts of cancer and had difficulty carrying out their daily responsibilities such as childcare. They described their pre-emptive worrying about the potential cancer diagnosis and recounted their thoughts about how it would negatively and significantly affect their obligations, responsibilities and lifestyle, citing examples such as their ability to be a good mother. For instance, a mother with a young child at home described the situation in this way: 
... I was worried; I have a seven-year-old boy and when you don't know... what's happening, you think about the worst-case scenario...cancer and then the treatment and how this is going to affect your lifestyle and the fact that you have a...very young child. I worry about the future, as a mother... Participant \#5.

These women, expecting the worst-case scenario, explained their experience prior to testing as being frozen by their fear and having difficulty functioning in their daily tasks.

Finally, one contextual similarity was found among the women of this category (above clinical cut-offs in both anxiety and uncertainty): their shared family history with cancer. They experienced the highest levels of distress, with scores up to 73 with a possible maximum score of 80 .

Three days Post-diagnosis - Receiving a cancer diagnosis

Among the group who received a cancer diagnosis (7/13), five had anxiety scores above the cut-off, and two for uncertainty. Three major themes were described by those receiving a cancer diagnosis: 1) as "lifechanging"; 2) as having to face many uncertainties and stressors, and 3) for two women, as a "relief".

Cancer is life-changing: A cancer diagnosis was described by these participants as life-changing with many uncertainties. One of the participants captured this feeling when she explained how she felt after hearing she has breast cancer, "I mean how can you not be upset, your whole life is completely discombobulated at that point because you have so many things to think about and your whole life is going to completely change Participant \#3." The women described feeling anxious about how they would be able to maintain their daily routines, for themselves and others, such as their weekly physical activity routine. Half (4/7) of the women described how overwhelmed they felt and how upset they were at their bodies for having let them down. They portrayed the situation as one of the most stressful events of their lives. Yet some women said that they felt thankful for the way the healthcare team attempted to reassure them, providing them with immediate details of treatment plans and that they felt supported as they moved into the next phase of being a cancer patient. In the days following the diagnosis, the women described how they reached out for support from family, with one specifying how she sought refuge in her religious faith.

Uncertainty and anxiety with the treatments and potential side effects: The uncertainty of their treatment plan and the unfamiliarity with potential side-effects left women feeling anxious, "...I have heard that you're really sick in chemo, but are you sick the whole time or are you just sick on certain days and then the whole idea of losing your hair (Participant \#3)." Another woman described how her treatment plan, which consisted of chemotherapy, was going to affect her ability to enjoy the seasons and holidays, "...I knew it was a year, a year by the time you go through all this (chemotherapy). So then in your mind, you're thinking, okay well I'm going to miss...the fall and Christmas. This isn't going to be great” (Participant \#8). To obtain their diagnosis, women went through additional testing such as biopsy. One woman described this experience as a funeral moment. 
A sense of relief: The sense of relief expressed by two women in the cancer group was described by three certainties "know[ing] what the [diagnosis] was Participant \#3", "knowing cancer had not metastasized (Participant \#8)", and "being recommended treatment that did not include mastectomy (Participant $\# 11)$ ". Knowing their breast abnormality was actually cancer provided a sense of certainty to some of the women. They now knew what they were dealing with (cancer) and were able to formulate treatment plans in line with their wishes. This, in turn, gave them a feeling of control over the situation. As described by one of the women, "When they recommended a lumpectomy...tsunami of relief rush[ed] through me because I was so afraid of losing my breast that when they said lumpectomy, I was like, fine... where do I sign? (Participant \#11)".

Three days post-diagnosis testing results - Receiving a benign diagnosis

Among the group who received a benign diagnosis (6/13), all had anxiety scores below cut-off with uncertainty remaining high for two. Two themes describe their experience at post-diagnosis for this subgroup: a) absolute relief and b) adjusting to the diagnosis.

Absolute relief: The six women described a sense of relief upon hearing that their suspicious breast lump was benign. The women described the event as a huge weight taken off their shoulders allowing them to return to their daily routine and habits, as well as validating their initial instincts that the lump would be found to be benign or their suspicions unfounded. One participant (Participant \#10), who had advocated for a referral to the RDC to receive additional testing to confirm a previous diagnosis of benign fibroadenoma, explained that she felt relief from having it "confirmed" by a biopsy.

Adjusting to the diagnosis: For two women with a benign diagnosis, uncertainty levels remained above normative values at T2 and for three women at T3. Despite their diagnosis, two of these women were scheduled to receive a lumpectomy and further pathological testing to remove abnormal tissue formation. They described the need for further investigation using language that was suggestive of residual uncertainty such as how tumour growth is unpredictable, and no diagnosis is ever $100 \%$ certain, thus warranting the removal of abnormal growth, as depicted in the following statements: "...we cannot predict every single detail of what's going to happen next, so...when I got the diagnosis that it's a great possibility that it's $100 \%$ benign but there is still a chance...(Participant \#4)" and "there is a possibility that it could be a type of benign tumour...that could get larger, so the recommendation is to have it removed (Participant \#7)."

Another participant who continued to present with residual uncertainty three days post-diagnosis despite receiving a benign diagnosis reported that she understood her diagnosis as having both a malignant and benign form of cancer that could later develop into full cancer.

Three weeks post-diagnosis - results for all

At three weeks of post-testing results, anxiety and uncertainty levels did not reveal many changes quantitatively except for an increase in those from the benign group with two now experiencing anxiety 
above the clinical cut-off. These two participants both described that they expect to eventually receive a cancer diagnosis. One woman describing her belief as "the negative now is that I am waiting for it...I missed this one....but I figure it's going to hit me at $60,65 . .$. so you know other women would say, oh my God it's a blessing, it's fantastic, I'm saved! No, I'm waiting. I'm waiting for the shoe to drop (Participant \#5)".

The Need for Support During the Diagnostic Period

The women expressed a need to receive details of the day's unfolding and especially what medical procedures were going to be done, how, and why, in advance of the day of testing. They wanted to know if they needed to take pain medication prior to coming to the clinic and if it was it best to be accompanied. On the day of receiving their results, half of the women would have wanted more information on their diagnosis such as a written report on the stage of their diagnosis. One woman suggested that a lecture on breast cancer diagnosis and the possible stages that might be found during the testing would help "as a way to brace [themselves]" (Participant \#8) for all diagnostic possibilities. The women recommended that they be asked if they would prefer to receive such a lecture while waiting to obtain further testing or while waiting for their results. Some of the women viewed the option of knowing in advance the possible implications of being diagnosed with cancer as a means by which to lower their uncertainties and distress.

In addition to their information needs, most of the women described a need for supportive care. The women described a need for some type of preparatory emotional support or counselling to help acquire and build adequate coping skills prior to attending the RDC. The women suggested having a one-time telephone call from a nurse before and after attending RDC to assess their support needs and coping skills. They also suggested having educational sessions on the upcoming procedures to reduce the uncertainties of the testing day's event.

\section{Discussion}

The study findings help address several current gaps in the empirical literature examining women's emotional experiences of having a suspicious breast lump requiring further investigation through RDC. As observed in this study, the women experienced high anxiety and uncertainty levels during the prediagnostic phase. These results accord with Mishel's uncertainty theory (1990), which explains that when faced with unfamiliarity, uncertainty arises. Thus, uncertainty theory would suggest that if women viewed the need for additional testing at the RDC as a threat rather than an opportunity, then their appraisal of their uncertain medical condition would increase their risk of developing further symptoms of psychological distress. In this study, the women faced the unfamiliarity of not knowing what to expect from their additional testing and facing a potential threat that could disrupt their daily lives. Being in an ill-defined situation, as explained by the uncertainty theory, further pulls women toward identifying the situation as a threat. When the event is viewed as a threat, there is a greater likelihood for an individual to experience higher levels of anxiety levels (Carleton et al., 2012). All of these components were likely to 
play a role in the women's emotional experience of undergoing further testing for a suspicious breast abnormality. One avenue of research to explore further would be methods to guide and support those going through RDC for suspicious breast abnormalities to assess their situation as an opportunity instead of a threat. Several examples of how the RDC experience could be framed as an opportunity include 1) an opportunity for quick screening; 2 ) an opportunity to spend fewer days living with the uncertainty of having cancer or not, and in some instances, 3) an opportunity to benefit from a quicker turnaround to begin treatment. By offering women optional perspectives by which to appraise their lifethreatening situation positively, such an approach could help women to view the uncertainties experienced during the waiting period and upon the receipt of their diagnosis, as opportunities for action planning.

The use of the mixed-methods approach in this study also led to further time-specific insights into the women's emotional experiences (29). The qualitative data helped to explain the women's reactions to the waiting period and the period following diagnosis. Within this group of women, a small group used positive reframing to keep their anxiety low while waiting to obtain further testing, while for others, the lack of understanding as to why the additional test was needed and how it would take place, made them feel even more anxious and experience high levels of uncertainty. Harnessing quantitative evidence of the women's emotional responses while also capturing the detailed nuances of their time-specific reactions via qualitative data, helped to achieve a more robust understanding of women's experiences of rapid testing for breast abnormalities than with quantitative data alone.

By focusing on the specific needs of each patient, nurses working in RDC can be invaluable in assisting women to cope with the uncertain experience and the highly anxious waiting period of rapid testing. Nurses can teach coping and relaxation skills such as positive reframing, facilitate communication with other professionals on the healthcare team and provide procedural support to help decrease short-term anxiety in women undergoing further cancer tests $(5,6,12,30,31)$. The integration of a nurse navigator in an RDC to reduce anxiety and increase satisfaction has been discussed positively in the literature $(12,32$, 33). Adapted education for RDCs with the support of a nurse navigator was reported as an important component to reducing distress and helping to women prepare for decision-making around treatment options (34). The findings from the current study offer further avenues to reduce anxiety and uncertainty prior to and after attending an RDC.

Implications for Practice

The majority of the women in our study experienced a considerable amount of uncertainty; all experienced heightened anxiety while waiting for further testing; and this experience impacted their daily personal, familial, and professional living activities. These results of high levels of anxiety accord with other studies investigating the emotional responses of women who received an abnormal screening mammography result and were waiting for further testing and diagnosis (35). For example, Pineault (35) noted that out of the 631 women who took part in their study, 51\% were quite or very anxious at every stage of the pre-diagnostic phase. Our results show $69 \%$ of the women reporting very high anxiety levels 
during the pre-diagnostic phase. Our findings also indicate that for those women scheduled for further investigation, screening for distress should be considered and seen as a way to predict and target those in greater need of support. The findings further underline the need for emotional support during the posttesting period, especially for those found to have cancer. This study also illustrated that at three-weeks post-testing, targeted psychosocial support may also be helpful to those with a benign diagnosis.

The women's suggestions for solutions provided several avenues for possible further support to women undertaking rapid diagnostic testing. One suggestion aligns with the idea of a nurse navigator who could be available to guide them through the day's process prior to and after testing was suggested. Several delivery options of this support may need to be explored, such as group teaching, telehealth or individual telephone support (33). Considering the short time frame between the discovery of a suspicious breast lump to the day of testing at the RDC, the latter two support options might be most feasible. Patient preferences and individual needs along with personnel availability may, in the end, dictate which approach would be most appropriate.

Recommendations for research

There remain outstanding gaps in our full understanding of the emotional impact and implications of attending RDC following the discovery of a suspicious breast abnormality. Notably, future studies are needed to investigate how psycho-educational nursing led-interventions during and after the diagnostic process can reduce anxiety and uncertainty and which interventions are effective and provide greatest satisfaction to patients. Such studies could provide guidance towards the development of new models of care for RDCs across Canada.

Study limitations

Like many other studies investigating the clinical and psychological impact of RDC (16), this study's findings are constrained by its sex selection bias and small sample size. This study was carried out in a cancer centre offering rapid diagnostic testing for women found with a suspicious breast lump. Yet, the strengths of the mixed-methods findings contribute significantly to our empirical understanding of the women's emotional experience attending an RDC, and the potential role a nurse that can play within this clinical context to address the full range of emotional and information needs across all diagnosis types.

\section{Conclusion}

RDCs offer women found with a suspicious breast lump the opportunity for quicker diagnostic testing. The short period between the discovery of a suspicious breast abnormality and further testing at an RDC is marked by high, intense, emotional turmoil. Anxiety and uncertainty levels remain high for the group with malignant tumour post-diagnosis and three weeks post-diagnosis. Considering the women's descriptions and the anxiety and uncertainty levels observed by the women who took part in this study, further investigation towards the possible provision of psychosocial support as part of the nurse working in RDC care is warranted. 


\section{Abbreviations}

SD - standard deviation

MUIS-C - Mishel Uncertainty in Illness Scale - Community form

RDC - Rapid diagnostic centres

SPSS - Statistical Package for the Social Sciences

STAI-S - Spielberger's State-Anxiety scale

\section{Declarations}

\section{Funding.}

$\mathrm{CM}$ received funding from the Reseau de recherche en interventions en sciences infirmières du Québec (RRISIQ) to conduct this study.

7. Authors' contributions.

Authors had full access to the data in the study and take responsibility for the integrity of the data and the accuracy of the data analysis. Conceptualization, C.M., D.H., B.L., D.M., A.T.; Methodology, D.H., C.M.; Investigation, D. H., B.L., C. M.; Formal Analysis, C.M., A T., B. .L.; Writing - Original Draft, all authors; Writing - Review and Editing, C. M., A. T., J. G.; Funding Acquisition, C. M.

8. Acknowledgments:

9. The authors would like to thank the study participants and the administration of the hospital where data collection took place. The author (CM) would like to acknowledge the support from The McGill Nursing Collaborative for Education and Innovation in Patient and family-centred Care (Newton Foundation/McGill Faculty of Medicine) and Graphos/McGill Writing Centre through the Writing for Publication initiative.

\section{References}

1.

Gurevich M, Devins GM, Wilson C, McCready D, Marmar CR, Rodin GM. Stress response syndromes in women undergoing mammography: a comparison of women with and without a history of breast cancer. Psychosom Med. 2004;66(1):104-12.

2.

Keyzer-Dekker CM, de Vries J, Mertens MC, Roukema JA, van der Steeg AF. The impact of diagnosis and trait anxiety on psychological distress in women with early stage breast cancer: a prospective study. $\mathrm{Br} \mathrm{J}$ Health Psychol. 2014;19(4):783-94.

3.

Liao MN, Chen MF, Chen SC, Chen PL. Uncertainty and anxiety during the diagnostic period for women with suspected breast cancer. Cancer nursing. 2008;31(4):274-83. 
4.

Liao MN, Chen PL, Chen MF, Chen SC, Chen YH. Supportive care for Taiwanese women with suspected breast cancer during the diagnostic period: effect on healthcare and support needs. Oncology nursing forum. 2009;36(5):585-92.

5.

Montgomery M, McCrone SH. Psychological distress associated with the diagnostic phase for suspected breast cancer: systematic review. J Adv Nurs. 2010;66(11):2372-90.

6.

Montgomery M. Uncertainty During Breast Diagnostic Evaluation: State of the Science. Oncology nursing forum. 2010;37(1):77.

7.

Pruett SB. Stress and the immune system. Pathophysiology. 2003;9(3):133-53.

8.

Gerra G, Monti D, Panerai AE, Sacerdote P, Anderlini R, Avanzini P, et al. Long-term immune-endocrine effects of bereavement: relationships with anxiety levels and mood. Psychiatry research. 2003;121(2):145.

9.

Stein M, Keller SE, Schleifer SJ. Stress and immunomodulation: the role of depression and neuroendocrine function. Journal of immunology (Baltimore Md: 1950). 1985;135(2 Suppl):827 s-33 s. 10.

Brocken P, Prins JB, Dekhuijzen PN, van der Heijden HF. The faster the better?-A systematic review on distress in the diagnostic phase of suspected cancer, and the influence of rapid diagnostic pathways. Psycho-oncology. 2012;21(1):1-10.

11.

Gagliardi A, Grunfeld E, Evans WK. Evaluation of diagnostic assessment units in oncology: a systematic review. Journal of clinical oncology: official journal of the American Society of Clinical Oncology. $2004 ; 22(6): 1126-35$.

12.

Singh M, Maheu C, Brady T, Farah R. The psychological impact of the rapid diagnostic centres in cancer screening: A systematic review. Canadian Oncology Nursing Journal/Revue Canadienne de Soins Infirmiers en Oncologie. 2017;27(4):348-55.

13.

McKevitt EC, Dingee CK, Leung SP, Brown CJ, Van Laeken NY, Lee R, et al. Reduced Time to Breast Cancer Diagnosis with Coordination of Radiological and Clinical Care. Cureus. 2017;9(12):e1919.

14.

Dey P, Bundred N, Gibbs A, Hopwood P, Baildam A, Boggis C, et al. Costs and benefits of a one stop clinic compared with a dedicated breast clinic: randomised controlled trial. BMJ. 2002;324(7336):507. 15.

Iwatani T, Matsuda A, Kawabata H, Miura D, Matsushima E. Predictive factors for psychological distress related to diagnosis of breast cancer. Psycho-oncology. 2013;22(3):523-9. 
16.

Morse JM, Pooler C, Vann-Ward T, Maddox LJ, Olausson JM, Madden C, et al. Awaiting diagnosis of breast cancer: Strategies of enduring for preserving self. Oncol Nurs Forum. 2014;41(4):350-9.

17.

Howard RA, Harvey PG. A Longitudinal Study of Psychological Distress in Women with Breast Symptoms. J Health Psychol. 1998;3(2):215-26.

18.

Mishel M. Uncertainty in illness. Image-the journal of nursing scholarship. 1988;20(4):225-32. 19.

Jensen D, Cohen JN, Mennin DS, Fresco DM, Heimberg RG. Clarifying the unique associations among intolerance of uncertainty, anxiety, and depression *. Cogn Behav Ther. 2016;45(6):431-44.

20.

Mishel MH. The measurement of uncertainty in illness. Nursing research. 1981;30(5):258-63.

21.

Mishel MH. Uncertainty in acute illness. Annu Rev Nurs Res. 1997;15:57-80.

22.

Bailey DE, Wallace M, Latini DM, Hegarty J, Carroll PR, Klein EA, et al. Measuring illness uncertainty in men undergoing active surveillance for prostate cancer. Applied nursing research: ANR. 2011;24(4):1939.

23.

Spielberger C, Buela-Casal G, Agudelo D, Carretero-Dios H, Santolaya F. Analysis of convergent and discriminant validity of the Spanish experimental version of the State-Trait Depression Questionnaire (STDEP). Actas espanolas de psiquiatria. 2005;33(6):374-82.

24.

Spielberger CD. Manual for the State-Trait Anxiety Inventory: STAI. In: Press CP, editor. Palo Alto: Consulting Psychologists Press; 1983.

25.

Knight RG, Waal-Manning HJ, Spears GF. Some norms and reliability data for the State-Trait Anxiety Inventory and the Zung Self-Rating Depression scale. The British journal of clinical psychology. 1983;22:245-9. ; (Pt 4 ).

26.

$\mathrm{UH}$

Graneheim

B-M

Lindgren

B

Lundman

2017

Graneheim UH, Lindgren B-M, Lundman B. Methodological challenges in qualitative content analysis: A discussion paper. Nurse Education Today. 2017;56:29-34. 
27.

Graneheim UH, Lundman B. Qualitative content analysis in nursing research: concepts, procedures and measures to achieve trustworthiness. Nurse Educ Today. 2004;24(2):105-12.

28.

Creswell JW, Plano Clark VL. Designing and conducting mixed methods research. Thousand Oaks: SAGE Publications; 2007.

29.

Hansen M, O’Brien K, Meckler G, Chang AM, Guise J-M. Understanding the value of mixed methods research: the Children's Safety Initiative-Emergency Medical Services. Emerg Med J. 2016;33(7):489-94. 30.

Carnwell R, Baker S. A. A patient-focused evaluation of breast care nursing specialist services in North Wales. Clinical Effectiveness in Nursing. 2003;7(1):18-29.

31.

Zanchetta Santos M, Maheu C, Baku L, Nembhard PJS, Lemonde M. Prospective roles for Canadian Oncology nurses in breast cancer rapid diagnostic clinics. Canadian Oncology Nursing Journal. 2015;25(2):144-9 (English). 50-56 (French).

32.

Ferrante J, Chen P-H, Kim S. The Effect of Patient Navigation on Time to Diagnosis, Anxiety, and Satisfaction in Urban Minority Women with Abnormal Mammograms: A Randomized Controlled Trial. J Urban Health. 2008;85(1):114-24.

33.

Liao MN, Chen PL, Chen MF, Chen SC. Effect of supportive care on the anxiety of women with suspected breast cancer. J Adv Nurs. 2010;66(1):49-59.

34.

Gilbert JE, Green E, Lankshear S, Hughes E, Burkoski V, Sawka C. Nurses as patient navigators in cancer diagnosis: review, consultation and model design Nurses as patient navigators in cancer diagnosis. Eur $\mathrm{J}$ Cancer Care. 2011;20(2):228-36.

35.

Pineault P. Breast Cancer Screening: Women's Experiences of Waiting for Further Testing. Oncol Nurs Forum. 2007;34(4):847-53.

\section{Methods}

The study setting was a rapid diagnostic breast centre (RDC) situated in large Canadian cancer research, treatment, and educational centre. The study followed a sequential mixed-method design. A quantitative phase followed by a qualitative phase was used to describe and understand the anxiety and uncertainty experienced by women undergoing rapid diagnostic testing for a suspicious breast abnormality. The quantitative data were collected at three time points (at pre-diagnosis (T1), three days post-diagnosis (T2), and three weeks post-diagnosis (T3)). The women's accounts from the qualitative data were collected three weeks post-testing, coinciding with the last time point measurement of quantitative data. 
The purpose was to deepen the understanding of the women's scores on the anxiety and uncertainty scales as experienced during the rapid diagnostic phase and to obtain their views on the type of support that might be needed during the diagnostic phase. Data collection occurred in 2012-2013. Research ethics approval was obtained from the Research Ethics Board of the participating hospital \#14-092-CE.

\section{Sampling}

Inclusion criteria included women from the hospital's catchment area, who had a suspicious breast abnormality that was detected radiologically or clinically, with no previous history of breast cancer diagnosis, who had an appointment at the RDC and who did not have a known BRCA1/BRCA2 mutation. Women were excluded if they did not read or speak English, were less than 18 years of age, had a recent fine needle aspiration that was suspicious for malignancy, or had a pre-existing diagnosis of anxiety, or major depressive disorder. A convenient sampling approach was used to select women. Women who met the inclusion criteria were identified and approached initially by the clinic's medical administrative assistant using an information script. If they were interested in participating, women were asked for verbal permission to have their name and telephone number forwarded to the study research assistant, who arranged for and conducted the full, informed consent process. Only those that provided written consent were included in the analysis. In total, 13 women met the eligibility criteria and agreed to participate in the study.

\section{Data Collection and Measures}

The quantitative data collected included demographics and two validated scales measuring uncertainty and anxiety. Uncertainty was measured using Mishel Uncertainty in Illness Scale - Community form (MUIS-C) (20). The MUIS-C is a 23 -item scale with responses rated on a 5-point Likert scale ranging from 23 to 115 with a mid-range score of 69 and normative values for breast cancer identified at 33.4 (20-22). In our study, alpha coefficients ranged from 0.88--0.97. Anxiety was measured using the Spielberger's State-Anxiety scale (STAI-S) $(23,24)$, a 20 -item scale with responses rated on a 4-point Likert scale ranging gram 20-80 with a study clinical cut-off for anxiety set at mid-range 40 (25). Alpha coefficients in our study ranged from 0.94 to 0.97 .

Questionnaires were administered over the telephone; the participant had a copy of the questionnaire to follow along while the research assistant read out the questions and answers. The questionnaires were administered at pre-diagnosis ( $\mathrm{T} 1$ ), three days post-diagnosis (T2), and three weeks post-diagnosis (T3). At T3, women were invited to participate in a telephone semi-structured interview that focused on 1) the participant's perception of the diagnostic process, 2) challenges faced during this process, 3 ) their views on areas of uncertainty and anxiety during this process, and 4) suggestions for service improvement. Examples of questions from the interview were: Tell me what it was like waiting for further testing and waiting for your results? What feelings or concerns did you have during the waiting times? Was waiting for your test results stressful for you? What was it like "not knowing"? What suggestions would you have to improve the diagnostic process? All participants were interviewed except one participant who was lost to follow-up following T2. All interviews were audio-recorded and transcribed verbatim. 


\section{Data Analysis}

Quantitative data: The statistical software SPSS 20 was used to generate percentages, means, standard deviations, and reliability scores (Cronbach's alpha), as well as non-parametric statistical analyses to test for significance and correlation.

Qualitative data: Qualitative data generated from the semi-structured interviews were transcribed verbatim and coded using content analysis $(26,27)$ to develop the categories and themes that represented the aggregate data. Three members $(\mathrm{CM}, \mathrm{CW}, \mathrm{DH})$ independently coded the data. Interview coding continued until a consensus of coding categories and thematic saturation were reached.

Triangulation of Quantitative and Qualitative Data: This study followed a mixed, quantitative-qualitative, sequential analytical approach in which the quantitative data was dominant and analyzed first, followed by the analysis of the qualitative data, used as an adjunct to further understand the quantitative findings (28). A matrix was developed to show high and low scores of uncertainty and anxiety with the qualitative data providing a deeper understanding of the emotion as experienced during and after testing. The same three investigators independently reviewed the integration of quantitative and qualitative data, and then met as a team to discuss the final findings until consensus was reached.

\section{Tables}

\begin{tabular}{|c|c|c|}
\hline $\begin{array}{l}\text { Timepoint } \\
\text { Measurement }\end{array}$ & Anxiety Above cut-off (40) & Uncertainty Above normative values BC (33.7) \\
\hline T1 (pre-diagnosis) & $\begin{array}{l}\text { Malignant } \mathrm{n}=3 \\
\text { Benign } \mathrm{n}=6 \\
\mathbf{n} \text { total }=\mathbf{9}\end{array}$ & $\begin{array}{l}\text { Malignant } \mathrm{n}=6 \\
\text { Benign } \mathrm{n}=7 \\
\mathrm{n} \text { total }=\mathbf{1 3}\end{array}$ \\
\hline T2 (3 days post-diagnosis) & $\begin{array}{l}\text { Malignant } \mathrm{n}=5 \\
\text { Benign } \mathrm{n}=0 \\
\mathbf{n} \text { total }=\mathbf{5}\end{array}$ & $\begin{array}{l}\text { Malignant } \mathrm{n}=2 \\
\text { Benign } \mathrm{n}=3 \\
\mathrm{n} \text { total }=\mathbf{6}\end{array}$ \\
\hline T3 (3 weeks post-diagnosis) & $\begin{array}{l}\text { Malignant } \mathrm{n}=3 \\
\text { Benign } \mathrm{n}=2 \\
\mathbf{n} \text { total }=\mathbf{5}\end{array}$ & $\begin{array}{l}\text { Malignant } \mathrm{n}=4 \\
\text { Benign } \mathrm{n}=3 \\
\mathrm{n} \text { total }=\mathbf{7}\end{array}$ \\
\hline
\end{tabular}

